\title{
A Survey of Methods for Deciding Whether a Reaction Network is Multistationary
}

\author{
B. $\operatorname{Joshi}^{1 *}$, A. Shiu ${ }^{2}$ \\ 1 Dept. of Mathematics, CSU San Marcos, 333 S. Twin Oaks Valley, San Marcos CA 92096, USA \\ 2 Dept. of Mathematics, Mailstop 3368, Texas A\&M University, College Station TX 77843-3368, USA
}

\begin{abstract}
Which reaction networks, when taken with mass-action kinetics, have the capacity for multiple steady states? There is no complete answer to this question, but over the last 40 years various criteria have been developed that can answer this question in certain cases. This work surveys these developments, with an emphasis on recent results that connect the capacity for multistationarity of one network to that of another. In this latter setting, we consider a network $N$ that is embedded in a larger network $G$, which means that $N$ is obtained from $G$ by removing some subsets of chemical species and reactions. This embedding relation is a significant generalization of the subnetwork relation. For arbitrary networks, it is not true that if $N$ is embedded in $G$, then the steady states of $N$ lift to $G$. Nonetheless, this does hold for certain classes of networks; one such class is that of fully open networks. This motivates the search for embedding-minimal multistationary networks: those networks which admit multiple steady states but no proper, embedded networks admit multiple steady states. We present results about such minimal networks, including several new constructions of infinite families of these networks.
\end{abstract}

Keywords and phrases: chemical reaction networks, mass-action kinetics, multiple steady states, deficiency, injectivity

Mathematics Subject Classification: 65H04, 37C25, 34A34, 70K42, 37C10, 80A30

\section{Introduction}

Reaction networks taken with mass-action kinetics arise in many scientific areas, from epidemiology (the SIR model) to population biology (Lotka-Volterra) to systems of chemical reactions. Indeed, reaction networks often form a suitable modeling framework when the variables of interest take non-negative values. In the resulting mathematical model, a unique positive stable steady state in the mathematical model can underlie robustness in the corresponding biological system; conversely, the existence of multiple positive stable steady states can explain switching behavior in the biological system.

This motivates the following important open question: which reaction networks, when taken with massaction kinetics, have the capacity for multistationarity? In other words, for which networks do there exist a choice of positive reaction rate constants and a choice of a forward-invariant set (or equivalently, a

*Corresponding author. E-mail: bjoshi@csusm.edu

(C) EDP Sciences, 2015 
choice of non-negative initial concentrations) within which the corresponding dynamical system admits two or more (nondegenerate) steady states?

At this time, there is no complete answer to this question; indeed, multistationary networks have not been completely catalogued. Nonetheless, over the last 40 years various criteria have been developed that can either preclude or guarantee multistationarity for certain classes of networks. The aim of the present article is to review these existing criteria, with an emphasis on recent results that connect the capacity for multistationarity of one network to that of certain related networks. We will take a broad view, instead of focusing on specific families arising in chemistry or biochemistry. For a historical survey of experimental findings concerning multistationarity, see the book of Marin and Yablonsky [34, Chapter 8].

Among the criteria mentioned above for precluding multistationarity are the injectivity criterion [8] and the deficiency zero and deficiency one theorems [19]. However, failing both the injectivity criterion and the conditions of the deficiency theorems is not sufficient for admitting multiple steady states. An instance of such a network is the following:

$$
B \leftrightarrows 0 \leftrightarrows A \quad 3 A+B \rightarrow 2 A+2 B
$$

(For readers familiar with the literature, this network has deficiency one, and each of the two linkage classes have deficiency zero, so neither the deficiency zero nor deficiency one theorems applies. Also, the injectivity criterion fails.) For this network, a simple calculation rules out multiple steady states, or the deficiency one algorithm can be applied to reach the same conclusion.

A somewhat more complicated example is the following:

$$
\begin{array}{cc}
0 \leftrightarrows A & 0 \leftrightarrows B \\
2 A \leftrightarrows A+B & A+C \leftrightarrows B+C
\end{array}
$$

In this case, the injectivity criterion fails, and the network has deficiency two - which implies that the deficiency theorems do not apply, nor does the deficiency one algorithm. Nevertheless, the advanced deficiency algorithm rules out multistationarity in this case.

By way of comparison, consider the next network:

$$
\begin{array}{ccr}
0 \leftrightarrows A & 0 \leftrightarrows B & 0 \leftrightarrows C \\
2 A \leftrightarrows A+B & A+B \leftrightarrows B+C
\end{array}
$$

where the only change from the previous network is that the complex $A+C$ in the last reaction has been replaced by the complex $A+B$. This time we find that the network does admit multiple steady states, because (1) it contains outflow reactions (such as $A \rightarrow 0$ ) for all three chemical species $(A, B, C)$, and (2) a so-called "CFSTR atom of multistationarity" network, namely, $\{0 \leftrightarrows A, 0 \leftrightarrows B, 0 \leftrightarrows C, B \rightarrow$ $A+B, 2 A \rightarrow A+B\}$ is "embedded" in the network (see Theorem 4.5 and Corollary 5.2).

Concerning more complicated networks, how can we assess multistationarity? In order to address this question, some recent work including our own has adopted the following strategy: instead of working with a specific network, can we relate the properties of two or more networks? Specifically, does a (large) network inherit some properties (such as multistationarity) of certain simpler related networks?

This question is intimately connected with and motivated by the problem of model choice (cf. [23]). A biological system displays a myriad of interactions among its components which take place at a wide variety of temporal scales. A good modeling design involves critical decisions about which components to incorporate within the model and which ones can be safely left out. In turn, any such decision about inclusion and exclusion of features is guided by the properties that the modeler is interested in examining as the model output, be it stability, multistationarity, oscillations, chaos, or some other behavior.

Within a cell, a huge array of chemical species might interact with each other in a variety of ways. In this setting, suppose a model consists of a set of chemical reactions including one of the form $A+B \rightarrow 2 C$; at least three major implicit assumptions typically underlie this choice of model: 
1. The presence or absence of other chemical species, besides $A, B$ and $C$, does not affect the rate of the reaction in which $A$ and $B$ combine to produce two molecules of $C$. However, future work may invalidate this assumption if further experimentation reveals that the reaction occurs only in the presence of an enzyme $E$, and thus the true chemical process is more accurately represented by one or more reactions involving $E$, such as $A+B+E \rightarrow 2 C+E$.

2. The chemical reactions that are included in the model are the only ones that are necessary to produce the dynamical range of behaviors under study, and inclusion of additional reactions will not significantly affect this dynamical repertoire. Of course, it may turn out that species $A$ participates in an as-yetunreported set of reactions involving a new substrate $F$, thus causing $A$ to be sequestered (and thus unavailable) for the original reaction $A+B \rightarrow 2 C$ when the substrate $F$ is introduced into the reaction vessel or cell.

3. At the time scale of interest, the reaction $A+B \rightarrow 2 C$ is a one-step reaction, in other words, there are no intermediate complexes that form as a molecule of $A$ combines with a molecule of $B$ to yield two molecules of $C$, and if any such intermediates do form, the time scale at which the intermediate species exist is much shorter than the time scale at which the species $A, B$, and $C$ are stable. For instance, the reaction $A+B \rightarrow 2 C$, when modeled at a finer temporal scale may be instead $A+B \leftrightarrows A B \rightarrow 2 C$.

Of course, assumptions such as these ones are unavoidable because biological processes tend to be quite complex, and even if the underlying mechanisms are known to a high level of detail, modeling every known feature is unrealistic. Indeed, underlying all modeling effort is an appeal to an implicit, redeeming principle that certain properties of the system are likely to be invariant to the level of detail included in the problem, once certain essential ingredients are incorporated into the model.

In this article, we explore the validity of this implicit assumption by probing it closely in the case of multistationarity of reaction networks: how accurately are the system dynamics reflected by our choice of which reactions, species, and complexes are included in the model? Can the number of steady states increase with the inclusion of more reactions or species? Can multistationarity be destroyed by increasing the level of detail included in the description of the model? Do the number of intermediate complexes (representing, for instance, various forms of an enzyme-substrate complex) in a model affect multistationarity? We take up these questions and describe some results in this direction.

This article is organized as follows. Section 2 introduces chemical reaction networks and the dynamical systems they define. Section 3 reviews existing criteria pertaining to the capacity of a given network for multistationarity. In Sections 4-5, we recall what it means for one network to be "embedded" in another, give some properties of this relation, and review existing criteria for when multiple steady states can be lifted from an embedded network. In Sections 6-7, we demonstrate the existence of infinitely many embedding-minimal multistationary and multistable networks. Finally, a discussion appears in Section 8 .

\section{Background}

In this section we recall how a chemical reaction network gives rise to a dynamical system.

We begin with an example of a chemical reaction: $A+B \rightarrow 3 A+C$. In this reaction, one unit of chemical species $A$ and one of $B$ react to form three units of $A$ and one of $C$. The reactant $A+B$ and the product $3 A+C$ are called complexes. The concentrations of the three species, denoted by $x_{A}, x_{B}$, and $x_{C}$, will change in time as the reaction occurs. Under the assumption of mass-action kinetics, species $A$ and $B$ react at a rate proportional to the product of their concentrations, where the proportionality constant is the reaction rate constant $\kappa$. Noting that the reaction yields a net change of two units in the amount of $A$, we obtain the first differential equation in the following system, and the other two arise 
similarly:

$$
\begin{aligned}
& \frac{d}{d t} x_{A}=2 \kappa x_{A} x_{B} \\
& \frac{d}{d t} x_{B}=-\kappa x_{A} x_{B} \\
& \frac{d}{d t} x_{C}=\kappa x_{A} x_{B} .
\end{aligned}
$$

A chemical reaction network consists of finitely many reactions. The mass-action differential equations that a network defines are comprised of a sum of the monomial contribution from the reactant of each chemical reaction in the network; these differential equations will be defined in equation (2.2).

\subsection{Chemical reaction systems}

We now provide precise definitions.

Definition 2.1. A chemical reaction network $G=(\mathcal{S}, \mathcal{C}, \mathcal{R})$ consists of three finite sets:

1. a set of chemical species $\mathcal{S}=\left\{A_{1}, A_{2}, \ldots, A_{s}\right\}$,

2. a set $\mathcal{C}=\left\{y_{1}, y_{2}, \ldots, y_{p}\right\}$ of complexes (finite nonnegative-integer combinations of the species), and

3. a set of reactions, which are ordered pairs of the complexes: $\mathcal{R} \subseteq \mathcal{C} \times \mathcal{C}$.

Throughout this work, the integer unknowns $p, s$, and $r$ denote the numbers of complexes, species, and reactions, respectively. Writing the $i$-th complex as $y_{i 1} A_{1}+y_{i 2} A_{2}+\cdots+y_{i s} A_{s}$ (where $y_{i j} \in \mathbb{Z}_{\geq 0}$ for $j=1,2, \ldots, s$ ), we introduce the following monomial:

$$
x^{y_{i}}:=x_{1}^{y_{i 1}} x_{2}^{y_{i 2}} \cdots x_{s}^{y_{i s}} .
$$

For example, the two complexes in the reaction $A+B \rightarrow 3 A+C$ considered earlier give rise to the monomials $x_{A} x_{B}$ and $x_{A}^{3} x_{C}$, which determine the vectors $y_{1}=(1,1,0)$ and $y_{2}=(3,0,1)$. These vectors define the rows of a $p \times s$-matrix of nonnegative integers, which we denote by $Y=\left(y_{i j}\right)$. Next, the unknowns $x_{1}, x_{2}, \ldots, x_{s}$ represent the concentrations of the $s$ species in the network, and we regard them as functions $x_{i}(t)$ of time $t$.

For a reaction $y_{i} \rightarrow y_{j}$ from the $i$-th complex to the $j$-th complex, the reaction vector $y_{j}-y_{i}$ encodes the net change in each species that results when the reaction takes place. We associate to each reaction a positive parameter $\kappa_{i j}$, the rate constant of the reaction. In this article, we will treat the rate constants $\kappa_{i j}$ as positive unknowns in order to analyze the entire family of dynamical systems that arise from a given network as the $\kappa_{i j}$ 's vary.

A network can be viewed as a directed graph whose nodes are complexes and whose edges correspond to the reactions. A linkage class is a connected component of the directed graph: the complexes $y$ and $y^{\prime}$ belong to the same linkage class if and only if there is a sequences of complexes $\left(y_{0}:=y, y_{1}, \ldots, y_{n-1}, y_{n}:=\right.$ $\left.y^{\prime}\right)$ such that either $y_{i} \rightarrow y_{i+1}$ or $y_{i+1} \rightarrow y_{i}$ is a reaction for all $0 \leq i \leq n-1$. A network is said to be weakly reversible if every connected component of the network is strongly connected. A reaction $y_{i} \rightarrow y_{j}$ is reversible if its reverse reaction $y_{j} \rightarrow y_{i}$ is also in $\mathcal{R}$; these reactions may be depicted as $y_{i} \rightleftharpoons y_{j}$.

The stoichiometric matrix $\Gamma$ is the $s \times r$ matrix whose $k$-th column is the reaction vector of the $k$-th reaction i.e., it is the vector $y_{j}-y_{i}$ if $k$ indexes the reaction $y_{i} \rightarrow y_{j}$. The reaction matrix is the negative of the transpose, $-\Gamma^{t} \in \mathbb{Z}^{r \times s}$, and the reactant (source) matrix is the $r \times s$ matrix whose $k$-th row is $y_{i}$ if $k$ indexes the reaction $y_{i} \rightarrow y_{j}$.

The choice of kinetics is encoded by a locally Lipschitz function $R: \mathbb{R}_{>0}^{s} \rightarrow \mathbb{R}^{r}$ that encodes the reaction rates of the $r$ reactions as functions of the $s$ species concentrations. The reaction kinetics system defined by a reaction network $G$ and reaction rate function $R$ is given by the following system of ODEs:

$$
\frac{d x}{d t}=\Gamma \cdot R(x)
$$


For mass-action kinetics, which is the setting of this paper, the coordinates of $R$ are $R_{k}(x)=\kappa_{i j} x^{y_{i}}$, if $k$ indexes the reaction $y_{i} \rightarrow y_{j}$. A chemical reaction system refers to the dynamical system (2.1) arising from a specific chemical reaction network $(\mathcal{S}, \mathcal{C}, \mathcal{R})$ and a choice of rate parameters $\left(\kappa_{i j}^{*}\right) \in \mathbb{R}_{>0}^{r}$ (recall that $r$ denotes the number of reactions) where the reaction rate function $R$ is that of mass-action kinetics. Specifically, the mass-action ODEs are the following:

$$
\frac{d x}{d t}=\sum_{y_{i} \rightarrow y_{j} \text { is in } \mathcal{R}} \kappa_{i j} x^{y_{i}}\left(y_{j}-y_{i}\right)=: \quad f_{\kappa}(x) .
$$

The stoichiometric subspace is the vector subspace of $\mathbb{R}^{s}$ spanned by the reaction vectors $y_{j}-y_{i}$, and we will denote this space by $S$ :

$$
S:=\mathbb{R}\left\{y_{j}-y_{i} \mid y_{i} \rightarrow y_{j} \text { is in } \mathcal{R}\right\} .
$$

Note that in the setting of $(2.1)$, one has $S=\operatorname{im}(\Gamma)$. For the network consisting of the single reaction $A+B \rightarrow 3 A+C$, we have $y_{2}-y_{1}=(2,-1,1)$, which means that with each occurrence of the reaction, two units of $A$ and one of $C$ are produced, while one unit of $B$ is consumed. This vector $(2,-1,1)$ spans the stoichiometric subspace $S$ for the network. Note that the vector $\frac{d x}{d t}$ in (2.1) lies in $S$ for all time $t$. In fact, a trajectory $x(t)$ beginning at a positive vector $x(0)=x^{0} \in \mathbb{R}_{>0}^{s}$ remains in the stoichiometric compatibility class, which we denote by

$$
\mathcal{P}:=\left(x^{0}+S\right) \cap \mathbb{R}_{\geq 0}^{s},
$$

for all positive time. In other words, this set is forward-invariant with respect to the dynamics (2.1).

A steady state of a reaction kinetics system (2.1) is a nonnegative concentration vector $x^{*} \in \mathbb{R}_{\geq 0}^{s}$ at which the ODEs (2.1) vanish: $f_{\kappa}\left(x^{*}\right)=0$. A steady state $x^{*}$ is nondegenerate if $\operatorname{Im}\left(d f_{\kappa}\left(x^{*}\right)\right) \stackrel{\geq 0}{=} S$. (Here, $d f_{\kappa}\left(x^{*}\right)$ is the Jacobian matrix of $f_{\kappa}$ at $x^{*}$.) A nondegenerate steady state is exponentially stable if each of the $\sigma:=\operatorname{dim} S$ nonzero eigenvalues of $d f_{\kappa}\left(x^{*}\right)$, viewed over the complex numbers, has negative real part. Also, we distinguish between positive steady states $x^{*} \in \mathbb{R}_{>0}^{s}$ and boundary steady states $x^{*} \in\left(\mathbb{R}_{\geq 0}^{s} \backslash \mathbb{R}_{>0}^{s}\right)$. A system is multistationary (or admits multiple steady states) if there exists a stoichiometric compatibility class $\mathcal{P}$ with two or more positive steady states. In the setting of mass-action kinetics, a network may admit multistationarity for all, some, or no choices of positive rate constants $\kappa_{i j}$; if such rate constants exist, then we say that the network itself is has the capacity for multistationarity or, for short, is multistationary. One focus of this work is on the following open question:

Problem 2.2. Which reaction networks are multistationary?

This question is difficult in general: assessing whether a given network is multistationary means determining if the parametrized family of polynomial systems arising from the mass-action ODEs (2.1) ever admits two or more positive solutions. Section 3 will describe results for certain families of networks, and Section 8 provides an outlook on future progress on this question.

\subsection{The deficiency of a chemical reaction network}

The deficiency $\delta$ of a reaction network is an important invariant. For a reaction network, recall that $p$ denotes the number of complexes. Also, let $l$ denote the number of linkage classes (connected components, as defined earlier). All networks considered in this article have the property that each linkage class contains a unique terminal strong linkage class, i.e. a maximal strongly connected subgraph in which there are no reactions from a complex in the subgraph to a complex outside the subgraph. In this case, Feinberg showed that the deficiency of the network can be computed in the following way:

$$
\delta:=p-l-\operatorname{dim}(S),
$$

where $S$ denotes the stoichiometric subspace (2.3). Note that in this case the deficiency depends only on the reaction network and not on the specific values of the rate constants. The deficiency of a reaction network is nonnegative because it can be interpreted as the dimension of a certain linear subspace [18]. 


\subsection{Networks with flow reactions}

Here we give some definitions pertaining to flow reactions (which in some settings may be interpreted as production and degradation).

Definition 2.3. 1. A flow reaction contains only one molecule; such a reaction is either an inflow reaction $0 \rightarrow X_{i}$ or an outflow reaction $X_{i} \rightarrow 0$. A non-flow reaction is any reaction that is not a flow reaction.

2. A reaction network is a continuous-flow stirred-tank reactor network (CFSTR network) if it contains outflow reactions $X_{i} \rightarrow 0$ for all species $X_{i}$ of the network.

3. A reaction network is fully open if it contains inflow reactions $0 \rightarrow X_{i}$ and outflow reactions $X_{i} \rightarrow 0$ for all species $X_{i}$ of the network. (Thus, a fully open network is a CFSTR.)

Notation. The subnetwork of a network $G$ consisting of the non-flow reactions of $G$ will be called the non-flow subnetwork of $G$ and denoted by $G^{\circ}$. Similarly, any reaction network $G=\{\mathcal{S}, \mathcal{C}, \mathcal{R}\}$ is contained in a fully open network obtained by including all flow reactions; we call this the fully open extension of $G$ and denote this CFSTR by

$$
\widetilde{G}:=\left\{\mathcal{S}, \mathcal{C} \cup \mathcal{S} \cup\{0\}, \mathcal{R} \cup\left\{X_{i} \leftrightarrow 0\right\}_{X_{i} \in \mathcal{S}}\right\}
$$

\section{Precluding or guaranteeing multiple steady states}

Broadly speaking, results for assessing multistationarity of a given network arise from two areas: deficiency theory and injectivity theory. This section surveys these results (see Table 1). Additionally, many recent results relate the capacity for multistationarity of one network to that of another; these will be described in more depth in Section 4.1. We note that, for simplicity, many of the results we describe are not stated here in their strongest or most general form (for instance, some results hold for more general kinetics than mass-action); instead, we refer the interested reader to the original papers.

TABLE 1. Results for precluding or guaranteeing multistationarity

\begin{tabular}{|l|l|l|l|}
\hline & $\begin{array}{l}\text { Precluding } \\
\text { multistationarity }\end{array}$ & $\begin{array}{l}\text { Guaranteeing } \\
\text { multistationarity }\end{array}$ & Both \\
\hline Deficiency theory & Theorems 3.1 and 3.2 & $\begin{array}{l}\text { Advanced deficiency and } \\
\text { higher deficiency theories }\end{array}$ \\
\hline Injectivity criterion & Theorems 3.3 and 4.16 & Theorem 4.17 & \\
\hline Using embedded networks & & & Theorem 4.5 \\
\hline Other approaches & Monotone systems results & & See Section 3.3 \\
\hline
\end{tabular}

\subsection{Deficiency theory}

Chemical reaction network theory, initiated by Feinberg, Horn, and Jackson beginning in the 1970s, aims to analyze reaction networks independently of the choice of rate constants. One key area of progress is deficiency theory. Here we review how these results can be used to preclude multistationarity. For details, we refer to the review paper of Feinberg $[19, \S 6]$. The following two results are due to Feinberg.

Theorem 3.1 (Deficiency zero theorem). Deficiency-zero networks are not multistationary. Moreover:

1. Every zero-deficiency network that is weakly reversible admits a unique positive steady state (for any choice of rate constants), and this steady state is locally asymptotically stable.

2. Every zero-deficiency network that is not weakly reversible admits no positive steady states (for any choice of rate constants). 
Theorem 3.2 (Deficiency one theorem). Consider a reaction network $G$ with linkage classes $G_{1}, G_{2}$, $\ldots, G_{l}$. Let $\delta$ denote the deficiency of $G$, and let $\delta_{i}$ denote the deficiency of $G_{i}$. Assume that:

1. each linkage class $G_{i}$ has only one terminal strong linkage class,

2. $\delta_{i} \leq 1$ for all $i=1,2, \ldots, l$, and

3. $\sum_{i=1}^{l} \delta_{i}=\delta$.

Then $G$ is not multistationary.

Additionally, Ellison, Feinberg, and Ji developed advanced deficiency and higher deficiency theories (including the deficiency one and advanced deficiency algorithms) which in many cases can affirm that a given network admits multiple steady states or can rule out the possibility [16, 20,29]. All of these deficiency-related results have been implemented in CRN Toolbox, freely available computer software developed by Feinberg, Ellison, Knight, and Ji [17]. For readers who have a particular network of interest of small to moderate size, we recommend exploring what the Toolbox can say about your network.

\subsection{The injectivity criterion}

In [8], Craciun and Feinberg introduced a criterion that guarantees that a reaction network does not admit multiple seady states. This test, which subsumed an earlier criterion of Schlosser and Feinberg [39], is applicable even when the deficiency zero and deficiency one theorems are not, so this approach is complementary to the results from deficiency theory.

The criterion arises from the following basic observation: letting $f_{\kappa}(x)$ denote the mass-action ODEs (2.2) arising from a network and rate constants $\kappa=\left(\kappa_{i j}\right)$, a sufficient condition to rule out multiple steady states is if the restricted map $\left.f_{\kappa}\right|_{\mathcal{P}}: \mathcal{P} \rightarrow S$ is injective for all $\kappa$ and all stoichiometric compatibility classes $\mathcal{P}$. Such a network is said to be injective or to pass the injectivity criterion (also called the Jacobian criterion).

The next result summarizes the contributions of many works that extended and gave equivalent formulations of the injectivity criterion. We let $\sigma(x) \in\{+,-, 0\}^{n}$ denote the sign vector of $x \in \mathbb{R}^{n}$. Also, for an $m \times n$ matrix $M$ and nonempty subsets $I \subseteq\{1,2, \ldots, m\}$ and $J \subseteq\{1,2, \ldots, n\}$, we let $M_{I, J}$ denote the submatrix of $M$ formed by the rows indexed by $I$ and columns indexed by $J$.

Theorem 3.3 (Injectivity criterion $[5,8-11,29,36,40,43])$. Let $G$ be a reaction network with stoichiometric matrix $\Gamma \in \mathbb{Z}^{s \times r}$, reactant matrix $M \in \mathbb{Z}_{>0}^{r \times s}$, and stoichiometric subspace $S=\operatorname{im}(\Gamma)$. Let $f_{\kappa}(x):=\Gamma \cdot R(x)$ denote the mass-action ODEs (2.2) arising from $G$ and rate constants $\kappa=\left(\kappa_{i j}\right) \in \mathbb{R}_{+}^{r}$. Then, the following are equivalent:

- For all $\kappa \in \mathbb{R}_{+}^{r}$ and all stoichiometric compatibility classes $\mathcal{P}$, the map $\left.f_{\kappa}\right|_{\mathcal{P}}: \mathcal{P} \rightarrow S$ is injective.

- For all $\kappa \in \mathbb{R}_{+}^{r}$ and all $x \in \mathbb{R}_{+}^{s}$, the Jacobian matrix of $f_{\kappa}(x)$ with respect to $x$ is injective on $S$.

- For all subsets $I \subseteq\{1,2, \ldots, s\}$ and $J \subseteq\{1,2, \ldots, r\}$ of size equal to the rank of $\Gamma$, the product $\operatorname{det}\left(\Gamma_{I, J}\right) \cdot \operatorname{det}\left(M_{J, I}\right)$ either is zero or has the same sign as all other nonzero such products, and moreover at least one such product is nonzero.

- The sets $\operatorname{ker}(\Gamma)$ and $M\left(\sigma^{-1}(\sigma(S))\right)$ have no nonzero sign vector in common.

If these equivalent conditions hold, then $G$ is not multistationary.

Remark 3.4. The original injectivity criterion of Craciun and Feinberg was for the case of CFSTRs (each chemical species has an associated outflow reaction) [8]. Another proof of their result appeared in the context of geometric modeling [12], and extended criteria were subsequently achieved for arbitrary networks $[22,25,29]$. For injectivity criteria for more general kinetics than mass-action, see the references listed in [36, Remark 3.5]. 
Remark 3.5. In some cases, the injectivity criterion can be translated to easy-to-check conditions on certain graphs arising from the network, namely, the species-reaction graph or the interaction graph $[3$, $10,27,35,41]$. We refer the interested reader to the review chapter of Craciun, Pantea, and Sontag [13].

Additionally, Ivanova obtained a result for precluding multistationarity that is based on the directed species-reaction graph [28]. More precisely, the graph yields a system of inequalities involving the rate constants that, if consistent, guarantees that the mass-action system avoids multiple steady states. For some networks, this inequality system is satisfied for all choices of rate constants, and thus multistationarity is precluded. For an example of such a network and an overview of these results, see [44, $\S 5.4]$.

Remark 3.6. Determining whether a given network is injective (and thus precludes multistationarity) can be accomplished easily with the online software tool CoNtRol [15] (which gives much more information as well). We encourage the reader to give it a try.

In their original setting of CFSTRs, Craciun and Feinberg extended ideas underlying Theorem 3.3 to give a criterion that can guarantee multiple steady states; we will postpone stating this result (namely, Theorem 4.17) until after introducing "embedded networks" in Section 4. A related result for guaranteeing multistationarity for non-injective networks appears in $[36, \S 3.2]$.

\subsection{Other approaches for assessing multistationarity}

Besides deficiency theory and injectivity theory, there are other approaches for determining whether a network is multistationary. We mention several here, and then in Section 4 describe another approach: relating the capacity for multiple steady states of two or more similar networks. First, monotone systems theory can be used to preclude multistationarity (for instance, see $[1,2,4,5,14]$ ). In this context, if a dynamical system (such as a chemical reaction system) preserves some partial order, then existence and uniqueness of a positive steady state can be guaranteed. For graph-theoretic criteria for monotonicity, see [13]. Additionally, Conradi and Mincheva used degree theory to obtain a graph-theoretic condition for ruling out multiple steady states which is applicable for systems for which trajectories are bounded [7]. Finally, Schlosser and Feinberg gave a condition guaranteeing multiple steady states for certain networks in which each linkage class consists of a pair of reversible reactions [39, Theorem 5.1].

\section{Precluding/guaranteeing multistationarity by using embedded networks}

In this section, we recall the definition of "embedding" and explain how this notion is useful for assessing multistationarity. Embedded networks were introduced by the authors in [31], generalizing Craciun and Feinberg's notion of network projections $[11, \S 8]$. The embedding relation generalizes the subnetwork relation - a subnetwork $N$ is obtained from a reaction network $G$ by removing a subset of reactions (alternatively, setting some of the reaction rates to be 0), while an embedded network is obtained by removing a subset of reactions or a subset of species (alternatively, setting the stoichiometric coefficients of those species to be 0 ) or both. For instance, removing the species $B$ from the reaction $A+B \rightarrow A+C$ results in the reaction $A \rightarrow A+C$.

In some cases, removing species results in a trivial reaction - where the source and product complex are identical. For instance, removal of both $B$ and $C$ from $A+B \rightarrow A+C$ results in the trivial reaction $A \rightarrow A$. So, after removing species, any trivial reactions and any copies of duplicate reactions are discarded.

Definition 4.1. Given a set of reactions $\mathcal{R}$ and a set of species $\mathcal{S}$, we define the restriction of $\mathcal{R}$ to $\mathcal{S}$, denoted $\left.\mathcal{R}\right|_{\mathcal{S}}$, to be the set of reactions obtained from $\mathcal{R}$ after implementing the following steps: (1) set the stoichiometric coefficients of species that are not in $\mathcal{S}$ equal to 0 , and then (2) discard any trivial reactions, that is, reactions of the form $C_{i} \rightarrow C_{i}$, where the source and product complexes are identical. 
As for removing both species and reactions, consider a network $G=(\mathcal{S}, \mathcal{C}, \mathcal{R})$ and a subset of reactions $\left\{y \rightarrow y^{\prime}\right\} \subseteq \mathcal{R}$ and a subset of species $\left\{X_{i}\right\} \subseteq \mathcal{S}$ that are to be removed. Then the reactions in the resulting embedded network $N$ will be denoted by $\mathcal{R}_{N}=\left.\left(\mathcal{R} \backslash\left\{y \rightarrow y^{\prime}\right\}\right)\right|_{\mathcal{S} \backslash\left\{X_{i}\right\}}$ in Definition $4.2-$ operationally, we obtain $N$ by first removing all the reactions $\left\{y \rightarrow y^{\prime}\right\}$ from $G$, and then removing the species $\left\{X_{i}\right\}$ from $G$, and finally restricting the set of reactions to the remaining species.

Definition 4.2. Let $G=(\mathcal{S}, \mathcal{C}, \mathcal{R})$. The embedded network $N$ of $G$ obtained by removing the set of reactions $\left\{y \rightarrow y^{\prime}\right\} \subseteq \mathcal{R}$ and the set of species $\left\{X_{i}\right\} \subseteq \mathcal{S}$ is

$$
N=\left(\left.\mathcal{S}\right|_{\mathcal{C}_{\mathcal{R}_{N}}},\left.\mathcal{C}\right|_{\mathcal{R}_{N}}, \mathcal{R}_{N}:=\left.\left(\mathcal{R} \backslash\left\{y \rightarrow y^{\prime}\right\}\right)\right|_{\mathcal{S} \backslash\left\{X_{i}\right\}}\right)
$$

Note that the subset of reactions that remain in the embedded network $N$ is not necessarily a complement of $\left.\left\{y \rightarrow y^{\prime}\right\}\right|_{\mathcal{S} \backslash\left\{X_{i}\right\}}$, because some reactions may be removed because they are either trivial reactions or duplicate reactions. Similarly, the subset of species that are in the embedded network need not be a complement of the subset of the removed species $\left\{X_{i}\right\}$. The following result establishes some basic properties about embedded networks; the proof appears in the Appendix.

Proposition 4.3. Consider networks $N$ and $G$, with stoichiometric subspaces $S_{N}$ and $S_{G}$ and deficiencies $\delta_{N}$ and $\delta_{G}$, respectively.

1. If $N$ is an embedded network of $G$, then $\operatorname{dim}\left(S_{N}\right) \leq \operatorname{dim}\left(S_{G}\right)$.

2. If $N$ is a subnetwork of $G$, and each linkage class of $N$ and each linkage class of $G$ contains a unique terminal strong linkage class, then $\delta_{N} \leq \delta_{G}$.

\subsection{Existing results for assessing multistationarity of one network from another}

In Section 3, we reviewed results for directly assessing multistationarity of a given network. In this section, we recall what is known about how the capacity for multistationarity of a given network $G$ is related to that of one of its embedded networks $N$. Most of these results "lift" steady states of $N$ to $G$, so in these settings ${ }^{1}$, if $N$ is multistationary then $G$ is too, or, equivalently, if $G$ is not multistationary then neither is $N$. Therefore these results can be used to preclude or to guarantee multistationarity. We summarize what is known in this setting in Theorem 4.5, of which parts 1 and 3 are due to the authors of the present work [32], part 2 is due to Craciun and Feinberg [9, Theorem 2], and part 4 is due to Feliu and Wiuf [23].

Definition 4.4. The induced network obtained by removing complexes $\left\{\mathcal{C}_{1}, \ldots, \mathcal{C}_{k}\right\}$ is obtained by removing those complexes and then replacing any pairs of reactions of the form $\mathcal{C}^{\prime} \rightarrow \mathcal{C}_{i} \rightarrow \mathcal{C}^{\prime \prime}$ with a reaction $\mathcal{C}^{\prime} \rightarrow \mathcal{C}^{\prime \prime}$ (and removing duplicate reactions, as necessary). Also, an intermediate complex has the form $X_{i}$, where $X_{i}$ is a species with total molecularity 1 (so it appears with stoichiometry 1 in the intermediate complex, and appears in no other complexes).

Theorem 4.5. Let $N$ and $G$ be reaction networks that are related in at least one of the following ways:

1. $N$ is a subnetwork of $G$ which has the same stoichiometric subspace as $G$,

2. $G$ is the fully open extension of $N$, i.e. $G=\widetilde{N}$ (in this case, $N$ is a subnetwork of $G$ ),

3. $N$ is a CFSTR embedded in a fully open network $G$,

4. $N$ is an induced network of $G$ obtained by removing one or more intermediates.

Then, if $N$ admits $m$ positive nondegenerate steady states (for some choice of rate constants), then $G$ admits at least $m$ positive nondegenerate steady states (for some choice of rate constants). Also, if $N$ admits $q$ positive, stable steady states, then $G$ admits at least $q$ positive, stable steady states.

\footnotetext{
${ }^{1}$ In general, it may be impossible to lift multiple steady states from an embedded network $N$ to $G$ [32, Example 4.5].
} 


$$
\text { "atoms-review" - 2015/8/22 - 20:07 — page } 56 \text { — \#10 }
$$

Remark 4.6. In the context of Theorem 4.5, $G$ might admit more steady states than $N$. For instance, a single reversible reaction has a unique positive steady state (by part 1 of the deficiency zero theorem), but making it irreversible yields no positive steady states (by part 2 of the theorem).

Remark 4.7. Each of the cases of Theorem 4.5 defines a partial order on the set of reaction networks. For case 4, Feliu and Wiuf called a minimal network with respect to this partial order (i.e. a network with no intermediates) a "core model", and then "extension models" are all networks obtained from the core model by adding intermediates. For case 3 (CFSTRs), the authors gave the name "CFSTR atoms of multistationarity" to the minimal networks [32] (see Definition 4.8 below), and now Theorem 4.5 motivates us to consider the general partial order formed by the embedding relation.

Definition 4.8. 1. A fully open network is a CFSTR atom of multistationarity if it is minimal with respect to the embedded network relation among all fully open networks that admit multiple nondegenerate positive steady states.

2. An embedding-minimal multistationary network is minimal with respect to the embedded network relation among all networks that admit multiple nondegenerate positive steady states.

3. A fully open network is a CFSTR atom of multistability if it is minimal with respect to the embedded network relation among all fully open networks that admit multiple nondegenerate positive stable steady states.

4. An embedding-minimal multistable network is minimal with respect to the embedded network relation among all networks that admit multiple nondegenerate positive stable steady states.

Note that any embedding-minimal multistationary network that is fully open is a CFSTR atom.

In Section 5, we will begin to answer the following open problems in the case of certain small networks:

1. Catalogue all CFSTR atoms of multistationarity.

2. Catalogue all embedding-minimal multistationary networks.

Remark 4.9. In order to implement Theorem 4.5, the authors propose the following "wish list":

1. A database of known CFSTR atoms of multistationarity, together with:

2. Software for automatically checking whether a given CFSTR contains as an embedded network a member of the database (which would imply that the larger CFSTR admits multiple steady states).

Going beyond item 1 of Theorem 4.5, we now consider the case that the stoichiometric subspaces of $G$ and its subnetwork $N$ do not coincide ${ }^{2}$. Then the next (easy) result states that a sufficient condition for being able to lift positive steady states (or for the larger network to have any positive steady states at all) is that some positive linear combination of the new reaction vectors must be in the stoichiometric subspace of $N$. In particular, adding a single reaction to $N$ that is not in the stoichiometric subspace of $N$ results in a network with no positive steady states.

Proposition 4.10. Let $N$ be a subnetwork of $G$ with subspaces $S_{N}$ and $S_{G}$, respectively. Let $T=\left\{y_{i} \rightarrow\right.$ $\left.y_{i}^{\prime}\right\}_{i=1, \ldots, q}$ denote the reactions in $G$ but not in $N$. If $S_{N} \neq S_{G}$ and no positive linear combination of the reaction vectors of $T$ is in $S_{N}$, i.e.

$$
\sum_{i=1}^{q} \alpha_{i}\left(y_{i}^{\prime}-y_{i}\right) \notin S_{N}
$$

for all $\left(\alpha_{i}\right) \in \mathbb{R}_{>0}^{q}$, then $G$ is not multistationary (and in fact $G$ has no positive steady states).

Proof. Let $\left\{y_{i} \rightarrow y_{i}^{\prime}\right\}_{i=q+1, \ldots, r}$ denote the reactions in $N$. If $x^{*}$ is a positive steady state of the massaction system arising from $G$ and rate constants $\kappa$, then letting $\alpha_{i}:=\kappa_{y_{i} \rightarrow y_{i}^{\prime}}\left(x^{*}\right)^{y_{i}}$ and rearranging the steady state equations yields $\sum_{i=1}^{q} \alpha_{i}\left(y_{i}^{\prime}-y_{i}\right)=\sum_{j=q+1}^{r} \alpha_{i}\left(y_{i}^{\prime}-y_{i}\right) \notin S_{N}$.

\footnotetext{
${ }^{2}$ In this case, when the two stoichiometric subspaces do not coincide, there is one result of the form in Theorem 4.5: Conradi et al. proved that under certain conditions one can lift multiple steady states from certain subnetworks called "elementary flux modes" [6, Supporting Information].
} 


$$
\text { "atoms-review" - 2015/8/22 - 20:07 - page 57 — \#11 }
$$

Remark 4.11. Underlying Proposition 4.10 and its proof is the following necessary condition for a network to admit positive steady states: the reaction vectors must be positively dependent $[20$, Remark 2.1 .

Remark 4.12. In Theorem 4.5, the number of steady states of a "smaller" network $N$ is a lower bound on the number for a "larger" network $G$. The only result known to us where (roughly speaking) a "smaller" network gives an upper bound on the number of steady states of a "larger" network is due to Feliu and Wiuf [23]. They considered the (larger) "extension models" of some "core model" network (recall Remark 4.7); then a "canonical model" is obtained by adding certain the reactions to the core model. If this canonical model is not multistationary, then every extension model of the core model also is not multistationary [23, Corollary 6.1].

\subsection{Using square embedded networks to apply the injectivity criterion to CFSTRs}

In Theorem 4.5, we saw that embedded networks are useful for both "ruling out" and "ruling in" multistationarity. In this section, we will see that certain embedded networks (namely, square embedded networks) can be used to elaborate on the injectivity criterion (Theorem 3.3) for the case of a CFSTR, and again give results both for precluding and guaranteeing multiple steady states (Theorems 4.16 and 4.17).

Definition 4.13. 1. A network is square if it has the same number of reactions and species.

2. Consider a square reaction network $G=(\mathcal{S}, \mathcal{C}, \mathcal{R})$ with $\mathcal{R}=\left\{y_{1} \rightarrow y_{1}^{\prime}, \ldots, y_{s} \rightarrow y_{s}^{\prime}\right\}$. The orientation of $G$ is $\operatorname{Or}(G)=\operatorname{det}\left(\left[y_{1}, \ldots, y_{s}\right]\right) \operatorname{det}\left(\left[y_{1}-y_{1}^{\prime}, \ldots, y_{s}-y_{s}^{\prime}\right]\right)$.

It is straightforward now (by translating part 3 of Theorem 3.3) to give the following equivalent formulation of the injectivity criterion: all square embedded networks of size equal to the dimension of the stoichiometric subspace and with nonzero orientation have the same orientation (and at least one is nonzero). We will state this in Theorem 4.16 for the case of CFSTRs.

Any square embedded network that contains an inflow reaction has zero orientation. On the other hand, removing a species which participates in an outflow reaction does not change the orientation of the resulting SEN. These two observations significantly simplify the calculation of orientation of all $s$-square networks of a CFSTR in $s$ species. In fact, we only need to examine the square embedded networks of $G^{\circ}$ where $G^{\circ}$ is the non-flow subnetwork of $G$. This is because there is an orientation-preserving one-to-one map between the subnetworks of $G$ containing $s$ reactions at least one of which is a non-flow reaction, and the square embedded networks of $G^{\circ}$.

We need the following definitions in order to state this result precisely.

Definition 4.14. Consider a reaction network whose set of non-flow reactions is given by:

$$
\begin{array}{rlrlrl}
y_{1} & \rightarrow y_{1}^{\prime} & y_{2} & \rightarrow y_{2}^{\prime} & \ldots & y_{l} \\
y_{l+1} & \leftrightarrow y_{l+1}^{\prime} & y_{l+2} & \leftrightarrow y_{l+2}^{\prime} \\
\prime & & & \ldots & y_{l+k} & \leftrightarrow y_{l+k}^{\prime},
\end{array}
$$

where none of the first $l$ reactions is reversible. The total molecularity of a species $X_{i}$ in the network is the following non-negative integer: $\operatorname{TM}\left(X_{i}\right)=\sum_{j=1}^{l+k}\left(y_{j i}+y_{j i}^{\prime}\right)$, where $y_{j i}$ is the stoichiometric coefficient of species $X_{i}$ in the complex $y_{j}$.

Definition 4.15. A network $N$ is relevant if it satisfies the following properties:

$-N$ has no outflows, inflows, generalized inflow reactions $0 \rightarrow \sum_{i} a_{i} X_{i}$, or generalized outflows $a X_{i} \rightarrow$ $b X_{i}$ (where $\left.0 \leq b \leq a\right)$.

$-N$ does not contain a pair of reversible reactions.

- Each species appears in at least two complexes and in at least one reactant complex.

- At least one species of $N$ has a total molecularity of at least 3 . 


$$
\text { "atoms-review" - 2015/8/22 - 20:07 - page 58 - \#12 }
$$

Non-relevant square embedded networks may be ignored for the purposes of establishing injectivity. This observation contributes to the following result:

Theorem 4.16 ([8], Theorems 4.9 and 5.1 of [31]). For a CFSTR $G$ with s species, with $G^{\circ}$ its subnetwork of non-flow reactions, the following are equivalent:

1. G passes the injectivity criterion (i.e. satisfies the equivalent conditions of Theorem 3.3).

2. Each s-square embedded network of $G$ has non-negative orientation.

3. Each square embedded network of $G^{\circ}$ has non-negative orientation.

4. Each relevant square embedded network of $G^{\circ}$ has non-negative orientation.

If these equivalent conditions hold, then $G$ is not multistationary.

The following is a partial generalization of Theorem 4.16: for any network $G$ (not necessarily a CFSTR) with stoichiometric subspace $S$, the network $G$ is injective if and only if each $(\operatorname{dim} S)$-square embedded network of $G$ has non-negative orientation. This result is simply a translation of item 3 of Theorem 3.3.

Finally, we present a criterion that can certify multistationarity in a CFSTR: if it fails the injectivity criterion (that is, the equivalent conditions in Theorem 4.16 do not hold), then sometimes a negatively oriented SEN can be used to guarantee multiple steady states. The result is due to Craciun and Feinberg.

Theorem 4.17 (Determinant optimization method, Theorem 4.2 of [8]). For a CFSTR G with s species, with $G^{\circ}$ its non-flow subnetwork, assume that there exists a negatively oriented square embedded network of $G^{\circ}$ of size $s$, consisting of reactions $y_{i} \rightarrow y_{i}^{\prime}$ (for $\left.i=1,2, \ldots, s\right)$, and that some positive linear combination of the negative reaction vectors $y_{i}-y_{i}^{\prime}$ is a positive vector (i.e. there exist $\eta_{1}, \eta_{2}, \ldots, \eta_{s}>0$ such that $\left.\sum_{i=1}^{s} \eta_{i}\left(y_{i}-y_{i}^{\prime}\right) \in \mathbb{R}_{>0}^{s}\right)$. Then, the fully open extension of $G$ is multistationary.

Building on Theorem 4.17, Feliu recently gave a new method based on injectivity for either guaranteeing or precluding multistationarity for general (not necessarily CFSTR) networks [21, §2.4].

\section{CFSTR atoms of multistationarity}

For certain small reaction networks, a complete characterization of multistationarity is known. Here we report on these results, including what is known about small CFSTR atoms of multistationary (recall Definition 4.8).

\subsection{Classification of multistationary, fully open networks with 1 non-flow reaction}

Fully open reaction networks with a single non-flow reaction (irreversible or reversible) form an infinite family of networks. Strikingly, however, the multistationary networks in this family have been completely determined. Moreover, their characterization depends entirely on a simple arithmetic relation on the stoichiometric coefficients, which is ascertained at a glance. The following result is due to the first author [30], and its proof uses primarily the deficiency one algorithm, along with deficiency theory (Theorems 3.1-3.2).

Theorem 5.1 (Classification of fully open networks with one non-flow reaction [30]). Let $n$ be a positive integer. Let $a_{1}, a_{2}, \ldots, a_{n}, b_{1}, b_{2}, \ldots, b_{n}$ be nonnegative integers.

1. The (general) fully open network with one irreversible non-flow reaction and $n$ species:

$$
\begin{aligned}
& 0 \leftrightarrows X_{1} \quad 0 \leftrightarrows X_{2} \quad \cdots \quad 0 \leftrightarrows X_{n} \\
& a_{1} X_{1}+\cdots+a_{n} X_{n} \rightarrow b_{1} X_{1}+\cdots+b_{n} X_{n}
\end{aligned}
$$

is multistationary if and only if $\sum_{i: b_{i}>a_{i}} a_{i}>1$. 
2. The (general) fully open network with one reversible non-flow reaction and $n$ species:

$$
\begin{aligned}
& 0 \leftrightarrows X_{1} \quad 0 \leftrightarrows X_{2} \quad \ldots \quad 0 \leftrightarrows X_{n} \\
& a_{1} X_{1}+\ldots a_{n} X_{n} \leftrightarrows b_{1} X_{1}+\ldots b_{n} X_{n}
\end{aligned}
$$

is multistationary if and only if $\sum_{i: b_{i}>a_{i}} a_{i}>1$ or $\sum_{i: a_{i}>b_{i}} b_{i}>1$.

Theorem 5.1 can be used to determine all CFSTR atoms with exactly one non-flow reaction:

Corollary 5.2 (Classification of CFSTR atoms of multistationarity with one non-flow reaction). Up to symmetry, the CFSTR atoms of multistationarity that have only one non-flow reaction are the following:

1. $\{0 \leftrightarrow A, m A \rightarrow n A\}$, where $m$ and $n$ are positive integers satisfying $n>m>1$.

2. $\{0 \leftrightarrow A, 0 \leftrightarrow B, A+B \rightarrow m A+n B\}$, where $m$ and $n$ are positive integers with $m>1$ and $n>1$.

In Section 6.1, we will see that all the CFSTR atoms in Corollary 5.2 are in fact embedding-minimal multistationary networks (Theorem 6.1).

\subsection{Classification of multistationary, fully open networks with 2 non-flow reactions}

A monomolecular complex has the form $X_{i}$ for some species $X_{i}$, while a bimolecular complex has the form $2 X_{i}$ or $X_{i}+X_{j}$. A complex is at most bimolecular if it is the zero complex, monomolecular, or bimolecular. A network is at most bimolecular if every complex in the network is at most bimolecular.

By Corollary 5.2, there are no at-most-bimolecular CFSTR atoms with only one non-flow reaction. An enumeration of at-most-bimolecular CFSTR atoms with two non-flow reactions (irreversible or reversible) was completed by the authors in [32]:

Theorem 5.3 (Classification of CFSTR atoms of multistationarity with two non-flow reactions and atmost-bimolecular complexes [32]). Up to symmetry, there are 11 CFSTR atoms of multistationarity that have only two non-flow reactions (irreversible or reversible) and complexes that are at most bimolecular:

1. $\{0 \leftrightarrows A, 0 \leftrightarrows B, A \rightarrow 2 A, A+B \rightarrow 0\}$

2. $\{0 \leftrightarrows A, 0 \leftrightarrows B, A \rightarrow 2 A, A \leftrightarrows 2 B\}$

3. $\{0 \leftrightarrows A, 0 \leftrightarrows B, 0 \leftrightarrows C, A \rightarrow 2 A, A \leftrightarrows B+C\}$

4. $\{0 \leftrightarrows A, 0 \leftrightarrows B, A \rightarrow A+B, 2 B \rightarrow A\}$

5. $\{0 \leftrightarrows A, 0 \leftrightarrows B, A \rightarrow A+B, 2 B \rightarrow 2 A\}$

6. $\{0 \leftrightarrows A, 0 \leftrightarrows B, A \rightarrow A+B \rightarrow 2 A\}$

7. $\{0 \leftrightarrows A, 0 \leftrightarrows B, A \rightarrow A+B, 2 B \rightarrow A+B\}$

8. $\{0 \leftrightarrows A, 0 \leftrightarrows B, B \rightarrow 2 A \rightarrow A+B\}$

9. $\{0 \leftrightarrows A, 0 \leftrightarrows B, B \rightarrow 2 A \rightarrow 2 B\}$

10. $\{0 \leftrightarrows A, 0 \leftrightarrows B, 0 \leftrightarrows C, A \rightarrow B+C \rightarrow 2 A\}$

11. $\{0 \leftrightarrows A, 0 \leftrightarrows B, A+B \rightarrow 2 A, A \rightarrow 2 B\}$

Thus, a CFSTR with two non-flow reactions (irreversible or reversible) admits multiple nondegenerate positive steady states if and only if it contains one of the eleven networks above as an embedded network.

Remark 5.4. The first network among the listed two-reaction CFSTR atoms $\{0 \leftrightarrows A, 0 \leftrightarrows B, A \rightarrow$ $2 A, A+B \rightarrow 0\}$, along with the one-reaction atom $\{0 \leftrightarrow A, 2 A \rightarrow 3 A\}$ have received close examination in the condensed matter theory literature because they exemplify important principles regarding multistationarity and systems that undergo phase transitions $[26,38]$. 


$$
\text { "atoms-review" — 2015/8/22 - 20:07 — page } 60-\# 14
$$

\section{Existence of infinitely many embedding-minimal multistationary networks}

Corollary 5.2 demonstrated that there are infinitely many CFSTR atoms of multistationarity with one non-flow reaction. In Section 6.1, we demonstrate that all the CFSTR atoms listed in that result are in fact embedding-minimal multistationary networks (Theorem 6.1), showing that there are infinitely many such networks. The molecularities of the complexes in those networks are arbitrarily large, which is unrealistic, so Section 6.2 introduces a family of "sequestration networks" which are conjectured in Section 6.3 to form an infinite family of networks with at-most-bimolecular complexes that are both CFSTR atoms and embedding-minimal multistationary networks.

\subsection{New result: two infinite families with one non-flow reaction}

Here we see that even among networks with only one species, there exist embedding-minimal multistationary networks. In fact, every CFSTR atom with only one non-flow reaction (enumerated earlier in Corollary 5.2) is an embedding-minimal multistationary network:

Theorem 6.1. For positive integers $m$ and $n$, consider the following networks: $G_{m, n}:=\{0 \leftrightarrow A, m A \rightarrow$ $n A\}$ and $H_{m, n}:=\{0 \leftrightarrow A, 0 \leftrightarrow B, A+B \rightarrow m A+n B\}$. Then

1. $G_{m, n}$ is a CFSTR atom of multistationarity and an embedding-minimal multistationary network if and only if $n>m>1$.

2. $H_{m, n}$ is a CFSTR atom of multistationarity and an embedding-minimal multistationary network if and only if $m>1$ and $n>1$.

Proof. That $G_{m, n}$ is multistationary if and only if $n>m>1$ follows from part 1 of Theorem 5.1. Additionally, those networks are CFSTR atoms of multistationarity (Corollary 5.2). Thus, it remains only to show that no proper embedded network of $G_{m, n}$ (when $n>m>1$ ) is multistationary. We begin by letting $s, l$, and $k$ denote the rates of the reactions $0 \rightarrow A, A \rightarrow 0$, and $m A \rightarrow n A$, respectively. Then, the single mass-action ODE (2.2) arising from $G_{m, n}$ is $\frac{d a}{d t}=s-l a+(n-m) k a^{m}$, where $a$ denotes the concentration of the species $A$; thus the number of positive steady states is the number of positive real roots of the univariate polynomial

$$
s-l a+(n-m) k a^{m} .
$$

The only nontrivial embedded networks of $G_{m, n}$ arise from removing reactions (as $G_{m, n}$ has only one species), i.e. setting one more of the reaction rates $s, l$, or $k$ to zero. It is straightforward to see that this results in the polynomial (6.1) having at most one positive real root.

By analogous reasoning for $H_{m, n}$, we need only show that no proper embedded network of $H_{m, n}$ (when $m, n>1$ ) is multistationary. We begin by denoting the rates of reactions $0 \rightarrow A, A \rightarrow 0,0 \rightarrow B, B \rightarrow 0$, and $A+B \rightarrow m A+n B$ by $s_{A}, l_{A}, s_{B}, l_{B}$, and $k$, respectively. Letting $a$ and $b$ represent concentrations of species $A$ and $B$, respectively, the mass-action mass-action ODEs (2.2) are the following:

$$
\begin{aligned}
& \frac{d a}{d t}=s_{A}-l_{A} a+(m-1) k a b \\
& \frac{d b}{d t}=s_{B}-l_{B} b+(n-1) k a b
\end{aligned}
$$

Now let $N$ be an embedded network of $H_{m, n}$. We first consider the case when at least one species (and possibly some reactions as well) are removed to obtain $N$.

(Species-removal case) If both species $A$ and $B$ are removed to obtain $N$, then $N$ is trivial. So, assume without loss of generality that species $B$ is removed. Thus, $N$ is a subnetwork of $\{0 \leftrightarrow A \rightarrow m A\}$, so its steady state equation is a linear equation in $a$, and thus cannot have multiple solutions. So, we may assume that no species are removed from $H_{m, n}$ to obtain $N$, i.e. $N$ is a subnetwork of $H_{m, n}$.

(Reaction-removal case) We must show that if $N$ is a proper subnetwork of $H_{m, n}$, i.e. if one or more rate constants is set to zero, then $N$ is not multistationary. If one of the outflow rates $l_{A}$ or $l_{B}$ is zero, then 


$$
\text { "atoms-review" — 2015/8/22 — 20:07 — page } 61 \text { — \#15 }
$$

there are no steady states as either $\dot{a}>0$ or $\dot{b}>0$ for all $(a, b)$ in the positive orthant. On the other hand, if $k=0$ or one of the inflow rates $s_{A}$ or $s_{B}$ is zero, then the steady state equations system can be solved explicitly, and there is at most one positive solution.

Remark 6.2. For the values of $m$ and $n$ for which $G_{m, n}$ or $H_{m, n}$ is multistationary, the parameter space (for the rate constants) for which there exist multiple positive steady states is identified in [30, Lemmas 4.3 and 4.5$]$.

The networks $G_{m, n}$ and $H_{m, n}$ have only one non-flow irreversible reaction, and only one species (in the case of $G_{m, n}$ ) or two species $\left(H_{m, n}\right)$. Both form infinite families, so the molecularities of the chemical species are unbounded. It may be argued that this is an unnatural property, and molecularity in realistic models must be small. In particular, if we restrict complexes to be at most bimolecular, then by Corollary 5.2 there are no CFSTR atoms of multistationarity that contain only one non-flow reaction (reversible or irreversible).

In Section 6.3, we will show that even with the bimolecular restriction there are infinitely many CFSTR atoms of multistationarity. Since molecularity is bounded, the numbers of reactions and species will be unbounded in this family. The demonstration is by explicit construction - using certain "sequestration networks" introduced next.

\subsection{Sequestration networks}

This subsection is a slight detour, in which we introduce sequestration networks, and apply many of our earlier results to study them. We will call reactions of the type $S+E \rightarrow 0$ sequestration reactions. Here we view $S$ as a substrate that binds with an enzyme $E$ and is then sequestered or rendered non-reactive. The reaction might be more accurately modeled as $S+E \rightarrow S E$, where $S E$ represents the substrateenzyme complex. However, we assume that the complex dissociates very rarely, and does not participate in any other reaction within the network, either because the complex is inert or because the complex leaves the reaction vessel through some unspecified mechanism. In such an event, it is reasonable to model the reaction, for simplicity and without changing the dynamics, as $S+E \rightarrow 0$.

Let us describe an instance of a sequestration reaction in Escherichia coli. The trp operon is a sequence of five genes that codes for the amino acid tryptophan. The regulatory protein, called trp repressor, can bind in the presence of tryptophan to the operator site of the trp operon and prevent its transcription. Thus, the presence of tryptophan inhibits its own production in E. coli [37] - we can model the process as a sequestration reaction with $E$ representing tryptophan and $S$ representing trp operon.

An even more compelling example is found in neuron signaling mechanisms. Communication between a pair of neurons occurs via the medium of neurotransmitters released by the presynaptic neuron into the synaptic cleft. The signal is terminated by the action of neurotransporters, which are neurotransmitter reuptake proteins that bind to neurotransmitter molecules and mediate their removal from the synaptic cleft [33]. In this case, $E$ represents neurotransporters and $S$ represents neurotransmitters. In general, even though an individual sequestration reaction is symmetric in $S$ and $E$, their distinct chemical roles are revealed when considered within a network.

Next we define a sequestration network, in which a synthesis reaction of the type $X_{1} \rightarrow m X_{n}$ is coupled with $n-1$ sequestration reactions. When $n$ is even, the sequestration reactions generate negative feedback to the synthesis reaction, while when $n$ is odd, the feedback to the synthesis reaction is positive. We will establish that this positive feedback results in the capacity for multiple steady states (Theorem 6.4). 


$$
\text { "atoms-review" — 2015/8/22 - 20:07 — page } 62 \text { — \#16 }
$$

Definition 6.3. For positive integers $n \geq 2, m \geq 1$, we define the sequestration network $K_{m, n}$ of order $n$ with production factor $m$ to be:

$$
\begin{aligned}
X_{1} & \rightarrow m X_{n} \\
X_{1}+X_{2} & \rightarrow 0 \\
\vdots & \\
X_{n-1}+X_{n} & \rightarrow 0
\end{aligned}
$$

The sequestration network $K_{m, n}$ has $n$ species, $n$ (non-flow) reactions, and for $1 \leq m \leq 2$, its complexes are at most bimolecular. Also, $K_{2, n}$ is an embedded network of, and is inspired by, a reaction network analyzed by Schlosser and Feinberg [39, Table 1].

The following result classifies the multistationarity of the fully open extensions of the $K_{m, n}$ 's; it will follow immediately from Lemmas 6.5 and 6.9 and Theorem 6.8 .

Theorem 6.4. For positive integers $n \geq 2$ and $m \geq 1$, the fully open extension $\widetilde{K}_{m, n}$ of the sequestration network $K_{m, n}$ is multistationary if and only if $m>1$ and $n$ is odd.

Our analysis of the sequestration networks begins with the following results.

Lemma 6.5. For any positive integer $n \geq 2$, if $m=1$, then the fully open extension $\widetilde{K}_{m, n}$ of the sequestration network $K_{m, n}$ is not multistationary.

Proof. For all $m \geq 1$, the maximum total molecularity of any species in $K_{m, n}$ is $m+1$. Thus, if $m=1$, then the maximum total molecularity is 2 , so by definition $K_{m, n}$ has no relevant square embedded networks. Therefore, by Theorem 4.16 the network does not admit multiple steady states.

Lemma 6.6. For positive integers $n \geq 2$ and $m \geq 1$, the sequestration network $K_{m, n}$ has no proper, relevant square embedded network.

Proof. Let $N$ be a relevant SEN of $K_{m, n}$. Then $N$ must contain the species $X_{n}$, because otherwise the maximum total molecularity of $N$ is less than three and $N$ must contain the reactions (6.2.1) and (6.2.n) because $X_{n}$ must appear in at least two complexes. $N$ must contain $X_{1}$ because otherwise (6.2.1) reduces to a generalized inflow reaction, which cannot occur in a relevant SEN. This further implies that $N$ must contain (6.2.2) because $X_{1}$ must occur in at least two complexes, which further implies that $X_{2}$ must be in $N$, because otherwise (6.2.2) reduces to an outflow reaction. Continuing this process, we find that the species $X_{1}, \ldots, X_{n}$ must be contained in $N$ and the reactions (6.2.1) to (6.2.n) must be in $N$. Thus $N=K_{m, n}$ is not a proper SEN of $K_{m, n}$.

Lemma 6.7. For positive integers $n \geq 2$ and $m \geq 1$, the sequestration network $K_{m, n}$ is negatively oriented if and only if $m \geq 2$ and $n$ is odd.

Proof. Write $K_{m, n}$ informally as $A_{n} \rightarrow B_{n}$, where $A_{n}$ is the source matrix and $B_{n}$ is the product matrix. Thus, the orientation $\operatorname{Or}\left(K_{m, n}\right)$ is the sign of the following:

$$
\operatorname{det} A_{n} \operatorname{det}\left(A_{n}-B_{n}\right):=\operatorname{det}\left(\begin{array}{cccccc}
1 & 0 & 0 & \ldots & 0 & 0 \\
1 & 1 & 0 & \ldots & 0 & 0 \\
0 & 1 & 1 & \ldots & 0 & 0 \\
\vdots & \vdots & \vdots & \ldots & \vdots & \vdots \\
0 & 0 & 0 & \ldots & 1 & 1
\end{array}\right) \operatorname{det}\left(\begin{array}{cccccc}
1 & 0 & 0 & \ldots & 0 & -m \\
1 & 1 & 0 & \ldots & 0 & 0 \\
0 & 1 & 1 & \ldots & 0 & 0 \\
\vdots & \vdots & \vdots & \ldots & \vdots & \vdots \\
0 & 0 & 0 & \ldots & 1 & 1
\end{array}\right)=1 \cdot\left(1+(-1)^{n+1}(-m)\right)
$$

which is negative if and only if $n$ is odd and $m \geq 2$. 


$$
\text { "atoms-review" — 2015/8/22 - 20:07 — page } 63 \text { — \#17 }
$$

Theorem 6.8. For positive integers $n \geq 2$ and $m \geq 2$, if $n$ is even, then the fully open extension $\widetilde{K}_{m, n}$ of the sequestration network $K_{m, n}$ is not multistationary. Furthermore, no sub-CFSTR (a subnetwork that is a CFSTR) of $\widetilde{K}_{m, n}$ is multistationary.

Proof. By Lemma $6.6, K_{m, n}$ has no proper, relevant SENs. Thus, the only relevant SEN, if $n$ is even, is $K_{m, n}$ itself, which is positively oriented by Lemma 6.7 . Thus, by Theorem $4.16, \widetilde{K}_{m, n}$ is not multistationary, nor is any subnetwork of $\widetilde{K}_{m, n}$ that is also a CFSTR (because relevant SENs of such a network are relevant SENs of $\left.K_{m, n}\right)$.

In the next section, we show that $\widetilde{K}_{m, n}$ is multistationary when $n$ is an odd.

\subsection{New result: an infinite family with complexes that are at most bimolecular}

Here we show that the fully open extension of the sequestration network $K_{m, n}$, which has $n$ reactions and $n$ species, admits multiple positive steady states when $n$ is odd.

Lemma 6.9. For positive integers $n \geq 2$ and $m \geq 2$, if $n$ is odd, then

1. $\widetilde{K}_{m, n}$ is a multistationary CFSTR, and

2. no fully open network that is an embedded network of $\widetilde{K}_{m, n}$ (besides $\widetilde{K}_{m, n}$ itself) is multistationary.

Proof. By Lemma 6.7 together with Theorem 4.17, to show that $\widetilde{K}_{m, n}$ is multistationary (for $m \geq 2$ and $n$ odd), we need only exhibit a positive linear combination of the reaction vectors $(1,0, \ldots, 0,-m),(1,1,0, \ldots, 0,0),(0,1,1,0 \ldots, 0,0), \ldots,(0,0, \ldots, 0,1,1)$ which is a positive vector. This can be achieved by choosing as coefficients $\left(\eta_{1}, \ldots, \eta_{n}\right)=(1,1, \ldots, 1, m+1)$.

For part 2 , let $N$ be a fully open network that is an embedded network of $\widetilde{K}_{m, n}$. Every SEN of the non-flow subnetwork $N^{\circ}$ of $N$ is an SEN of $K_{m, n}$, so in light of Theorem 4.16 and Lemmas 6.6 and 6.7, $N^{\circ}$ must equal $K_{m, n}$ in order for $N$ to be multistationary, i.e. $N=\widetilde{K}_{m, n}$.

The following conjecture is what remains for us to show that the networks $\widetilde{K}_{m, n}$ in Lemma 6.9 are CFSTR atoms of multistationarity. It is true for $n=3$ and $m=2$.

Conjecture 6.10. For positive integers $n \geq 2$ and $m \geq 2$, if $n$ is odd, then $\widetilde{K}_{m, n}$ admits multiple nondegenerate steady states.

Theorem 6.11. If Conjecture 6.10 holds, then:

1. For positive integers $n \geq 2$ and $m \geq 2$, if $n$ is odd, then $\widetilde{K}_{m, n}$ is a CFSTR atom of multistationarity.

2. There exist infinitely many embedding-minimal multistationary networks with complexes that are at most bimolecular.

Proof. Assume that Conjecture 6.10 holds. Then part 1 follows from part 2 of Lemma 6.9.

It follows that the $\widetilde{K}_{2, n}$ 's, for odd $n \geq 2$, form an infinite family of at-most-bimolecular CFSTR atoms of multistationarity. By definition, each such $\widetilde{K}_{2, n}$ has at least one subnetwork $N=N_{2, n}$ which is an embedding-minimal multistationary network.

We now claim that $K_{2, n}$ is a subnetwork of $N$. By way of contradiction, assume that this is not the case, so $N^{\circ}$, the non-flow subnetwork of $N$, is a proper, embedded network of $K_{2, n}$. Consider a square embedded network $N_{i}$ of $N$. If $N_{i}$ contains an inflow reaction, then its orientation is zero. On the other hand, if $N_{i}$ contains an outflow reaction, the species in the outflow reaction can be removed without changing the orientation of $N_{i}$. Thus, to establish injectivity of $N$, it suffices to show that all SENs of $N^{\circ}$ have nonnegative orientation (recall the discussion after Definition 4.13), which is furthermore equivalent to showing that all relevant SENs of $N^{\circ}$ have nonnegative orientation. Indeed, this follows from Lemma 6.6, as no proper SEN of $K_{2, n}$ is relevant.

Thus, $N_{2, n}$ has $n$ species, so these embedding-minimal multistationary networks (for odd $n$ ) form an infinite family. 


$$
\text { "atoms-review" - 2015/8/22 - 20:07 - page 64 — \#18 }
$$

\section{Existence of infinitely many embedding-minimal multistable networks}

In this section, we demonstrate the existence of infinitely many embedding-minimal multistable networks, again via explicit construction. We will build on the findings for the networks $G_{m, n}$ studied earlier.

Theorem 7.1. For positive integers $m \neq n$, consider the network: $\bar{G}_{m, n}:=\{0 \leftrightarrow A, m A \leftrightarrows n A\}$. Then $\bar{G}_{m, n}$ is a CFSTR atom of multistability and an embedding-minimal multistable network if and only if $n>1$ and $m>1$.

Proof. We assume without loss of generality that $n>m$. We know from Theorem 5.1 that $\bar{G}_{m, n}$ is multistationary if and only if $n>m>1$. Let $s, l, k_{+}$and $k_{-}$denote the rates of the reactions $0 \rightarrow A$, $A \rightarrow 0, m A \rightarrow n A$, and $n A \rightarrow m A$, respectively. Then, the single mass-action ODE (2.2) arising from $\bar{G}_{m, n}$ is $\dot{a}=s-l a+(n-m) k_{+} a^{m}-(n-m) k_{-} a^{n}$, where $a$ denotes the concentration of the species $A$; thus the number of positive steady states is the number of positive real roots of the univariate polynomial

$$
f(a):=s-l a+(n-m) k_{+} a^{m}-(n-m) k_{-} a^{n} .
$$

Note that $\bar{G}_{m, n}$ reduces to $G_{m, n}$ when $k_{-}=0$. By Theorem 5.1 , there exist parameter values $s, l, k_{+}>0$ for which $G_{m, n}$ has two nondegenerate positive steady states. Thus, for fixed $s, l, k_{+}>0$ such that $G_{m, n}$ has two nondegenerate positive steady states, there exists a sufficiently small $k_{-}>0$ such that $\bar{G}_{m, n}$ has three nondegenerate positive steady states. Furthermore, since $f(0)=s>0$ and $\lim _{a \rightarrow \infty} f(a)=-\infty$, the graph of $f(a)$ crosses the $a$-axis from above twice - these crossing points correspond to stable steady states.

The only nontrivial embedded networks of $\bar{G}_{m, n}$ arise from removing reactions (as $G_{m, n}$ has only one species), i.e. setting one of the reaction rates $s, l, k_{+}$or $k_{-}$to zero. It is straightforward to see that this results in the polynomial (7.1) having at most two positive real roots, of which at most one corresponds to a stable steady state.

\section{Discussion}

As described in the Introduction, deciding whether a given network arising in practice is multistationary is an important first step in understanding its dynamics. Here we have presented various criteria for answering this question. However, there are many networks for which none of the existing criteria apply. To this end, we have seen that the approach via "transferring" multistationarity from one network to a (typically larger) network can be helpful. Nonetheless, we are still far from a complete answer.

Looking forward, we highlight some future directions:

1. Future investigations will likely develop additional criteria for deciding multistationarity tailored to networks arising in specific application domains.

2. Some networks arising in biology are CFSTRs. Can we use the idea of CFSTR atoms or embeddingminimal multistationary networks to understand such networks that are multistationary? As an example, Fouchet and Regoes analyzed a multistationary CFSTR arising in immunology [24]; can we enumerate, and then interpret, the multistationary networks embedded in this network? Siegel-Gaskins et al. perfomed a related analysis of small gene regulatory networks [42]; see also [32, Remark 3.3].

3. We must address computational challenges inherent in enumerating CFSTR atoms and embeddingminimal multistationary networks, and then providing a method for determining whether a given network contains as an embedded network, a CFSTR atom, for instance.

Finally, our true interest is in atoms of multistability, as steady states observed in practice are necessarily stable. Therefore, we need more criteria, guided by networks arising in practice, for when such atoms can be lifted. 


\section{Appendix: properties of the embedded-network relation}

Here we prove Proposition 4.3, restated here as Proposition 8.2.

Lemma 8.1. Let $N$ be an embedded network of $G$.

1. If $N$ is a subnetwork of $G$, then the stoichiometric subspace of $N$ is a subspace of the stoichiometric subspace of $G$.

2. If $N$ is obtained by removing a set of species of $G$, then the stoichiometric subspace of $N$ is a projection of the stoichiometric subspace of $G$.

3. The number of complexes in $N$ is no more than the number of complexes in $G$.

Proof. The stoichiometric subspace of a network $N$ is $S_{N}:=\operatorname{span}\left\{y^{\prime}-y \mid y \rightarrow y^{\prime} \in N\right\}$. If $N$ is a subnetwork of $G,\left\{y^{\prime}-y \mid y \rightarrow y^{\prime} \in N\right\} \subseteq\left\{y^{\prime}-y \mid y \rightarrow y^{\prime} \in G\right\}$, and so $S_{N}$ is a subspace of $S_{G}$. If $N$ is obtained by removing a subset of species of $G$, the reaction vectors of $N$ are a projection of the reaction vectors of $G$, from which the second assertion follows. As for the third item, if $N$ is a subnetwork of $G$, then the complexes of $N$ are a subset of the complexes of $G$. In the case of species removal, every complex in $N$ is a projection of a complex in $G$, so this completes the proof.

Proposition 8.2. 1. If $N$ is an embedded network of $G$, the dimension of the stoichiometric subspace of $N$ is less than or equal to the dimension of the stochiometric subspace of $G$, $\operatorname{dim}\left(S_{N}\right) \leq \operatorname{dim}\left(S_{G}\right)$.

2. If $N$ is a subnetwork of $G$, then the deficiency of $N$ is no greater than the deficiency of $G$, that is, $\delta_{N} \leq \delta_{G}$.

Proof. The first assertion is a direction consequence Lemma 8.1. For the second item, we need only show that if $N$ is obtained from $G$ by removing one reaction, then $\delta_{N} \leq \delta_{G}$. In fact, we will show in this case that $\delta_{N} \in\left\{\delta_{G}, \delta_{G}-1\right\}$. Suppose that $N=G \backslash\left\{y \rightarrow y^{\prime}\right\}$, i.e. $\left\{y \rightarrow y^{\prime}\right\}$ is the reaction that is removed from $G$ to obtain $N$. If $y^{\prime}-y$ is in the span of the reaction vectors of $N$, then $\operatorname{dim}\left(S_{N}\right)=\operatorname{dim}\left(S_{G}\right)$, otherwise $\operatorname{dim}\left(S_{N}\right)=\operatorname{dim}\left(S_{G}\right)-1$. Let $p_{N}$ and $p_{G}$ represent the number of complexes, and let $l_{N}$ and $l_{G}$ represent the number of linkage classes of $N$ and $G$, respectively.

Case (i) $\left(p_{N}=p_{G}-2\right)$ : Removing the single reaction $\left\{y \rightarrow y^{\prime}\right\}$ from $G$ results in removing two complexes only if neither $y$ nor $y^{\prime}$ participates in any reaction of $N$. In other words, this case occurs only if $\left\{y \rightarrow y^{\prime}\right\}$ is itself a linkage class. Therefore, $l_{N}=l_{G}-1$, and the result follows.

Case (ii) $\left(p_{N}=p_{G}-1\right)$ : In this case, either $y$ or $y^{\prime}$, but not both of them, participates in at least one reaction of $N$, which implies that $l_{N}=l_{G}$.

Case (iii) $\left(p_{N}=p_{G}\right)$ : In this case, each of $y$ and $y^{\prime}$ participates in at least one reaction of $N$. Suppose that removal of $y \rightarrow y^{\prime}$ from $G$ results in a linkage class of $G$ splitting into two linkage classes of $N$, so that $l_{N}=l_{G}+1$. Then $\delta_{N} \in\left\{\delta_{G}, \delta_{G}-1\right\}$. If removal of $y \rightarrow y^{\prime}$ from $G$ does not split a linkage class, then $l_{N}=l_{G}$. Since the linkage class does not split, there exists a set of complexes in $N,\left\{y_{0}, y_{1}, \ldots y_{m-1}, y_{m}\right\}$ where $y_{0}:=y$ and $y_{m}:=y^{\prime}$ such that either $y_{i} \rightarrow y_{i+1}$ or $y_{i+1} \rightarrow y_{i}$ is a reaction in $N$ for all $i$ for which $0 \leq i \leq m-1$. But this implies that the reaction vector $y^{\prime}-y$ is a linear combination of the reaction vectors $\pm\left(y_{i}-y_{i+1}\right)$. Thus, $\operatorname{dim}\left(S_{N}\right)=\operatorname{dim}\left(S_{G}\right)$, and so $\delta_{N}=\delta_{G}$.

Remark 8.3. Removing a species from a reaction network, even if doing so does not decrease the number of reactions, can cause the deficiency to increase, decrease, or remain the same. For instance, removing species $A$ from $\{A+B \rightarrow 0, A \rightarrow 2 B\}$ yields the embedded network $\{B \rightarrow 0 \rightarrow 2 B\}$ and increases the deficiency from 0 to 1 . An example in which the deficiency does not change arises by removing $A$ from the reaction $A \rightarrow B$; both have deficiency 0. Finally, removing the species $A$ from the network $\{A+B \rightarrow C \rightarrow B \rightarrow D \rightarrow 2 A+B\}$ yields the network $\{C \leftrightarrows B \leftrightarrows D\}$, which decreases the deficiency from 1 to 0 .

Acknowledgements. AS was supported by the NSF (DMS-1312473). 


\section{References}

[1] D. Angeli, P. De Leenheer, E. Sontag. Graph-theoretic characterizations of monotonicity of chemical networks in reaction coordinates. J. Math. Biol., 61 (2010), no. 4, 581-616.

[2] M. Banaji. Monotonicity in chemical reaction systems. Dyn. Syst., 24 (2009), no. 1, 1-30.

[3] M. Banaji, G. Craciun. Graph-theoretic criteria for injectivity and unique equilibria in general chemical reaction systems. Adv. Appl. Math., 44 (2010), no. 2, 168-184.

[4] M. Banaji, J. Mierczyński. Global convergence in systems of differential equations arising from chemical reaction networks. J. Differential Equations, 254 (2013), no. 3, 1359-1374.

[5] M. Banaji, C. Pantea. Some results on injectivity and multistationarity in chemical reaction networks. preprint, http://arXiv.org/abs/1309.6771, (2013).

[6] C. Conradi, D. Flockerzi, J. Raisch, J. Stelling. Subnetwork analysis reveals dynamic features of complex (bio)chemical networks. Proc. Natl. Acad. Sci. USA, 104 (2007), no. 49, 19175-19180.

[7] C. Conradi, M. Mincheva. Graph-theoretic analysis of multistationarity using degree theory. preprint, http://arxiv.org/abs/1411.2896, (2014).

[8] G. Craciun, M. Feinberg. Multiple equilibria in complex chemical reaction networks. I. The injectivity property. SIAM J. Appl. Math., 65 (2005), no. 5, 1526-1546.

[9] G. Craciun, M. Feinberg. Multiple equilibria in complex chemical reaction networks: extensions to entrapped species models. IEE Proceedings-Systems Biology, 153 (2006), 179-186.

[10] G. Craciun, M. Feinberg. Multiple equilibria in complex chemical reaction networks. II. The species-reaction graph. SIAM J. Appl. Math., 66 (2006), no. 4, 1321-1338.

[11] G. Craciun, M. Feinberg. Multiple equilibria in complex chemical reaction networks: Semiopen mass action systems. SIAM J. Appl. Math., 70 (2010), no. 6, 1859-1877.

[12] G. Craciun, L. Garcia-Puente, F. Sottile. Some geometrical aspects of control points for toric patches. In M. Dæhlen M. S. Floater, T. Lyche, J.-L. Merrien, K. Morken, L. L. Schumaker, eds., Mathematical Methods for Curves and Surfaces, vol. 5862 of Lecture Notes in Comput. Sci., pages 111-135. Springer, Heidelberg, 2010.

[13] G. Craciun, C. Pantea, E. Sontag. Graph-theoretic characterizations of multistability and monotonicity for biochemical reaction networks, pages 63-72. Springer, 2011.

[14] P. Donnell, M. Banaji. Local and global stability of equilibria for a class of chemical reaction networks. SIAM J. Appl. Dyn. Syst., 12 (2013), no. 2, 899-920.

[15] P. Donnell, M. Banaji, A. Marginean, C. Pantea. CoNtRol: an open source framework for the analysis of chemical reaction networks. Bioinformatics, (2014), to appear.

[16] P. Ellison. The advanced deficiency algorithm and its applications to mechanism discrimination. Ph.D. thesis, University of Rochester, 1998.

[17] P. Ellison, M. Feinberg, H. Ji, D. Knight. Chemical Reaction Network Toolbox, 2011. Available at http://www.crnt. osu. edu/CRNTWin.

[18] M. Feinberg. Complex balancing in general kinetic systems. Arch. Rational Mech. Anal., 49 (1972), no. 3, $187-194$.

[19] M. Feinberg. Chemical reaction network structure and the stability of complex isothermal reactors I. The deficiency zero and deficiency one theorems. Chem. Eng. Sci., 42 (1987), no. 10, 2229-2268.

[20] M. Feinberg. Multiple steady states for chemical reaction networks of deficiency one. Arch. Rational Mech. Anal., 132 (1995), no. 4, 371-406.

[21] E. Feliu. Injectivity, multiple zeros, and multistationarity in reaction networks. preprint, http://arXiv.org/abs/1407.2955, (2014).

[22] E. Feliu, C. Wiuf. Preclusion of switch behavior in reaction networks with mass-action kinetics. Appl. Math. Comput. 219 (2012), 1449-1467.

[23] E. Feliu, C. Wiuf. Simplifying biochemical models with intermediate species. J. R. Soc. Interface, 10 (2013), no. 87.

[24] D. Fouchet, R. Regoes. A population dynamics analysis of the interaction between adaptive regulatory $T$ cells and antigen presenting cells. PLoS ONE, 3 (2008), no. 5, e2306.

[25] G. Gnacadja. A Jacobian criterion for the simultaneous injectivity on positive variables of linearly parameterized polynomials maps. Linear Algebra Appl., 437 (2012), 612-622.

[26] P. Grassberger. On phase transitions in Schlögl's second model. Zeitschrift für Physik B Condensed Matter, 47 (1982), no. $4,365-374$.

[27] J. W. Helton, I. Klep, R. Gomez. Determinant expansions of signed matrices and of certain Jacobians. SIAM J. Matrix Anal. Appl., 31 (2009), no. 2, 732-754.

[28] A. Ivanova. Conditions for uniqueness of stationary state of kinetic systems related to structural scheme of reactions. Kinet. Katal., 20 (1979), 1019-1023.

[29] H. Ji. Uniqueness of Equilibria for Complex Chemical Reaction Networks. Ph.D. thesis, Ohio State University, 2011.

[30] B. Joshi. Complete characterization by multistationarity of fully open networks with one non-flow reaction. Applied Mathematics and Computation, 219 (2013), 6931-6945.

[31] B. Joshi, A. Shiu. Simplifying the Jacobian criterion for precluding multistationarity in chemical reaction networks. SIAM J. Appl. Math., 72 (2012), no. 3, 857-876.

[32] B. Joshi, A. Shiu. Atoms of multistationarity in chemical reaction networks. Journal of Mathematical Chemistry, 51 (2013), no. 1, 153-178.

[33] K. P. Lesch, D. Bengel. Neurotransmitter reuptake mechanisms. CNS drugs, 4 (1995), no. 4, 302-322. 
[34] G. Marin, G. S. Yablonsky. Kinetics of Chemical Reactions. Wiley-VCH, Wienheim, Germany, 2011.

[35] M. Mincheva, G. Craciun. Multigraph conditions for multistability, oscillations and pattern formation in biochemical reaction networks. Proceedings of the IEEE, 96 (2008), no. 8, 1281-1291.

[36] S. Müller, E. Feliu, G. Regensburger, C. Conradi, A. Shiu, A. Dickenstein. Sign conditions for injectivity of generalized polynomial maps with applications to chemical reaction networks and real algebraic geometry. to appear in Found. Comput. Math.

[37] M. Santillán, M. C. Mackey. Dynamic regulation of the tryptophan operon: a modeling study and comparison with experimental data. Proceedings of the National Academy of Sciences, 98 (2001), no. 4, 1364-1369.

[38] F. Schlögl. Chemical reaction models for non-equilibrium phase transitions. Zeitschrift für Physik, 253 (1972), no. 2, $147-161$.

[39] P. M. Schlosser, M. Feinberg. A theory of multiple steady states in isothermal homogeneous CFSTRs with many reactions. Chemical Engineering Science, 49 (1994), no. 11, 1749-1767.

[40] G. Shinar, M. Feinberg. Concordant chemical reaction networks. Math. Biosci., 240 (2012), no. 2, 92-113.

[41] G. Shinar, M. Feinberg. Concordant chemical reaction networks and the species-reaction graph. Math. Biosci., 241 (2013), no. 1, 1-23.

[42] D. Siegal-Gaskins, M. K. Mejia-Guerra, G. D. Smith, E. Grotewold. Emergence of switch-like behavior in a large family of simple biochemical networks. PLoS Comput. Biol., 7 (2011), no. 5, e1002039.

[43] C. Wiuf, E. Feliu. Power-law kinetics and determinant criteria for the preclusion of multistationarity in networks of interacting species. SIAM J. Appl. Dyn. Syst., 12 (2013), 1685-1721.

[44] G. Yablonskii, V. Bykov, A. Gorban, V. Elokhin. Kinetic Models of Catalytic Reactions. Comprehensive Chemical Kinetics, vol. 32, ed. by R.G. Compton, Elsevier, Amsterdam, 1991. 